Open Access

\title{
Potential risk of residual cancer cells in the surgical treatment of initially unresectable pancreatic carcinoma after chemoradiotherapy
}

\author{
Hironobu Takano*, Takahiro Tsuchikawa, Toru Nakamura, Keisuke Okamura, Toshiaki Shichinohe and Satoshi Hirano
}

\begin{abstract}
Background: With development of chemoradiotherapy for pancreatic carcinoma, borderline resectable or initially unresectable cases sometimes become operable after long-term intensive chemoradiotherapy. However, there is no established strategy for adjuvant surgery with respect to whether the surgical resection should be extensive or downsized accordingly with diminished disease areas following response to chemoradiotherapy.

Methods: The clinical and pathological aspects of 18 patients with initially unresectable pancreatic cancer who underwent adjuvant surgery after chemo(radio)therapy in our department from 2007 were evaluated.

Results: Overall survival from initial treatment was much better for patients with RO resection than for patients with R1/2 resection. In two of three patients who had complete improvement of plexus (PL) invasion after chemo(radio)therapy, there had still remained pathological plexus invasion. It was shown that tumors did not shrink continuously from the tumor front, but parts remained discontinuously at the distal portion in the process of tumor regression by chemo(radio)therapy.

Conclusions: In adjuvant surgery for patients with locally advanced pancreatic cancer, the potential risk of residual cancer in the regression area following chemoradiotherapy should be considered. Achieving R0 resection will lead to an improved prognosis, and it is necessary to consider how well the extent of resection is after a favorable response to chemoradiotherapy.
\end{abstract}

Keywords: Initially unresectable pancreatic carcinoma, Chemoradiotherapy, Adjuvant surgery

\section{Background}

Pancreatic carcinoma is a disease with a very poor prognosis. Although surgery is the only curative option for this disease, only $20 \%$ of patients can be treated surgically [1]. The prognosis of patients with unresectable disease is significantly worse than that of patients with resectable disease. In the past, various treatments for unresectable pancreatic carcinoma had been adopted, but prognosis in patients with unresectable pancreatic carcinoma continues to be disappointing $[2,3]$. Recently, with the development of chemoradiotherapy for pancreatic carcinoma, "borderline resectable" or "initially unresectable" cases sometimes become operable after long-term, intensive chemoradiotherapy $[4,5]$. We have previously reported the outcomes

\footnotetext{
*Correspondence: htakano1231@yahoo.co.jp

Department of Gastroenterological Surgery II, Hokkaido University Graduate School of Medicine, N-15 W-7, Sapporo, Hokkaido 060-8638, Japan
}

of adjuvant surgery for initially unresectable pancreatic carcinoma with long-term chemoradiotherapy [6]. We defined "adjuvant surgery" as radical surgery for initially unresectable cases of pancreatic carcinoma which had a well response to chemo(radio)therapy without original intent to proceed to resection. There are several reports showing a correlation between prognosis and neural invasion or histopathological grading after neoadjuvant chemoradiotherapy of patients with pancreatic carcinoma $[7,8]$. However, there is no established strategy for "adjuvant surgery" with respect to whether the surgery should be extensive or whether the surgical resection could be downsized to correspond to the diminished disease areas following response to chemoradiotherapy.

Our surgical strategy for initially unresectable patients with a long-term favorable response to chemo(radio)therapy included the resection area initially affected by 
the tumor, because it was unknown whether cancer still remained or not in the area where suspected involved lesion had improved on imaging with good response to chemo(radio)therapy. In this report, the suitability of our surgical strategy for initially unresectable pancreatic carcinoma following chemoradiotherapy was evaluated based on the pathological analysis of the resected specimens.

\section{Methods}

\section{Patients}

From July 2007 to April 2013, surgery was performed following chemo(radio)therapy for 18 patients with initially unresectable pancreatic carcinoma in our department. Surgical indications for resectable tumors were determined based on the following criteria: no distant metastasis, no extension to the common hepatic artery (CHA) or the superior mesenteric artery (SMA) (head of the pancreas), and no extension to the gastro-duodenal artery (GDA) or the SMA (body and tail of the pancreas). For borderline resectable tumors, the surgical indications were based on the following criteria: no distant metastasis and extension to half around plexus invasion of the SMA. Other tumors were deemed unresectable. Patients with unresectable tumors subsequently underwent chemo(radio)therapy. Resectability of the tumors was determined by imaging and intraoperative findings and then radical surgery was performed, in principle, six or more months after the start of the initial treatment. Imaging diagnosis was done by a radiologist, surgeon, gastroenterologist, and an echo technician at a Cancer Board conference. In this study, all 18 patients were initially diagnosed unresectable tumors through this process. At the adjuvant surgery, our operative procedure included the resection area initially affected by the tumor. This study was approved by the Institutional Ethics Committee of Hokkaido University Hospital.

\section{Assessment of clinical and pathological efficacies after chemoradiotherapy}

Radiological assessment after initial treatment was performed according to Response Evaluation Criteria In Solid Tumors (RECIST) [9]. The pathological effect of preoperative therapy was assessed by Evans grading system [10]. These assessments were done by a radiologist, surgeon, gastroenterologist, pathologist, and an echo technician at a Cancer Board conference and at a clinicopathological conference, respectively.

\section{Survival analysis}

Overall patient survival was calculated from the date of initial treatment to the date of last follow-up (censored) or the date of patient death (event). Differences in survival times between patient subgroups were analyzed using the log-rank test. Survival probabilities were calculated using the Kaplan-Meier method. In all tests, statistical significance was set at $P<0.05$. All analyses were performed using $\mathrm{JMP}^{\circledR} 10$ software.

\section{Results}

\section{Patients' characteristics}

Patients' data and the perioperative data are shown in Tables 1 and 2. The median age of the 18 patients was 63 years (range: 43-68 years). The numbers of males and females were nearly equal. The tumor location was the head of the pancreas in 7 patients and the pancreatic body or tail in 11 patients. Histological tumor types of all patients before starting treatment were invasive ductal adenocarcinoma. Tumors were initially unresectable due to distant metastasis (hepatic metastasis or para-aortic lymph node metastasis) in 5 patients, para-aortic lymph node metastasis in 2 , hepatic metastasis in 3 , and major artery involvement in 13 patients. Of them, celiac artery invasion in 1, GDA invasion in 4, CHA invasion in 4, SMA invasion in 3, and portal vein (PV) tumor thrombus in 1 patient, respectively. We regarded these 13 patients who had invasion to major vessels and had no distant metastasis as the locally advanced cases. All patients were initially given chemo(radio)therapy. The treatment regimens are shown in Table 3. All 18 patients underwent chemotherapy including gemcitabine (GEM); 4 patients were treated with chemoradiotherapy. The median period for preoperative therapy was 9 months (range: 6-44 months).

\section{Surgical procedures performed}

Subtotal stomach-preserving pancreaticoduodenectomy (SSPPD) was performed for 7 patients, distal pancreatectomy with en bloc celiac axis resection (DP-CAR) was

\section{Table 1 Patient characteristics}

\begin{tabular}{lll}
\hline Characteristics & & $2007-2013 n=18$ \\
\hline Age (years) & Male & $63(43-68)$ \\
Sex & Female & 8 \\
& Ph & 10 \\
Tumor location & Pb & 7 \\
& Pt & 8 \\
Reason for initially unresectable & Locally advanced & 13 \\
& metastatic & 5 \\
Preoperative therapy & Chemo (IV) & 11 \\
& Chemo (TA) & 3 \\
RECIST & Chemoradi & 4 \\
& SD & 5 \\
& PR & 13 \\
& CR & 0
\end{tabular}

$P h$ pancreas head, $P b$ pancreas body, $P t$ pancreas tail, $S D$ stable disease, $P R$ partial response, $C R$ complete response 
Table 2 Perioperative data

\begin{tabular}{lll}
\hline Postoperative date & & $2007-2013 n=18$ \\
\hline Operative procedure & SSPPD & 7 \\
& DP-CAR & 7 \\
& DP & 3 \\
Op-CAR & 1 \\
Blood loss (ml) & & $418(200-879)$ \\
Complication & Total & $905(330-3200)$ \\
& Pancreatic fistula & $11(61 \%)$ \\
& SSI & $4(22 \%)$ \\
Hospital stay (days) & Ischemic gastritis & $1(22 \%)$ \\
Mortality & & $30(12-97)$ \\
Evans grade & & 0 \\
& I & 4 \\
& Ila & 6 \\
Residual tumor & IIb & 5 \\
& III & 1 \\
& IV & 2 \\
Ro & R1 & 15 \\
\hline R2 & 2 \\
\hline
\end{tabular}

SSPPD subtotal stomach-preserving pancreaticoduodenectomy, DP-CAR distal pancreatectomy with en bloc celiac axis resection, $D P$ distal pancreatectomy, $T P-C A R$ total pancreatectomy with en bloc celiac axis resection

performed for 7 patients, total pancreatectomy with en bloc celiac axis resection (TP-CAR) was performed for 1 patient, and distal pancreatectomy (DP) was performed for 3 patients. SSPPD was performed using the modified Child method. Concomitant vascular resection was basically performed if main vascular invasion or plexus invasion was suspected, except for the SMA. The SMA nerve plexus was removed circumferentially from the root of the SMA longitudinally along to the branch of the inferior pancreaticoduodenal artery in DP-CAR. Among the 18 patients, there were patients with main vessel resection and reconstruction including PV resection

Table 3 Neoadjuvant therapy

\begin{tabular}{llll}
\hline Chemo(radio)therapy & Regimen & $n$ & Period of therapy (month) \\
\hline Chemotherapy (IV) & GEM + S-1 & 10 & $8.5(5-32)$ \\
& GEM & 1 & 11 \\
Chemotherapy (TA) & GEM + 5-FU & 3 & $7 / 34 / 44$ \\
Chemoradiotherapy & GEM + S-1 & 2 & $7 / 16$ \\
& GEM + 5-FU & 1 & 8 \\
& GEM & 1 & 32 \\
& & & $9(5-44)$ \\
\hline
\end{tabular}

GEM gemcitabine, 5-FU 5-fluorouracil and reconstruction for 12 patients and arterial resection and reconstruction for 4 patients.

Among the three patients with hepatic metastasis, two patients initially had solitary metastasis in segment III and segment IV, respectively. The other patient had hepatic metastasis both in segment I and segment VIII, resulted in no pathological evidence of malignancy followed by concomitant partial hepatic resection. Previous two patients with solitary hepatic metastasis underwent resection for primary lesion alone, because the hepatic lesion was not identified in CT or completely shrunken before surgery.

\section{Objective tumor response to chemoradiotherapy}

R0 resection was achieved in 15 patients (83\%), while $\mathrm{R} 1$ resection was achieved in 2 patients (12\%) and R2 resection was achieved in 1 patient (5\%). The reasons for $\mathrm{R} 1$ resection were a pathologically positive diagnosis at the PV stump and a pathologically positive diagnosis at the arterial plexus around the SMA, respectively. And the reason for $\mathrm{R} 2$ resection was peritoneal metastasis identified just before abdominal closure.

Using Evans grading system for tumor response, 4 patients had grade I, 6 patients had grade IIa, 5 patients had grade IIb, 1 patient had grade III, and 2 patients had grade IV. Based on RECIST, 13 patients had partial response (PR), and 5 patients had stable disease (SD). No patients had complete response (CR), but 2 patients had pathological CR.

\section{Improvement of arterial plexus invasion on CT imaging over time}

Of the 18 patients, 12 patients (66\%) were initially diagnosed as having unresectable disease with arterial plexus invasion around the $\mathrm{CA}$ in 1 patient, the GDA in 4 patients, the SMA in 3 patients with pancreatic body cancer, and the $\mathrm{CHA}$ in 4 patients with head of the pancreas cancer. After the chemoradiotherapy, these findings on CT imaging had improved with the plexus around the artery of all patients. These fields, initially considered to include cancer cells, were totally resected in an en bloc-wise manner using already described procedures. Among them, 2 out of 3 patients in complete improvement after chemoradiotherapy and 7 out of 9 patients in partial improvement had still remained pathological plexus invasion (Table 4). Here, we show one characteristic case (no. 10 in Table 4) that was initially diagnosed as having unresectable disease with invasion to the plexus around the SMA. After chemotherapy including GEM and TS-1 for 15 months, adjuvant surgery was performed because PR was achieved (Fig. 1). CT scan after chemotherapy showed tumor shrinkage, which was amenable to radical resection by DP. However, DP-CAR was performed because of the policy described above. The 
Table 4 Data of patients with initially unresectable due to arterial plexus invasion

\begin{tabular}{|c|c|c|c|c|c|c|c|c|}
\hline No. & $\begin{array}{l}\text { Tumor } \\
\text { location }\end{array}$ & $\begin{array}{l}\text { Suspected arterial } \\
\text { plexus invasion }\end{array}$ & $\begin{array}{l}\text { Operative } \\
\text { procedure }\end{array}$ & $\begin{array}{l}\text { Combined } \\
\text { resection }\end{array}$ & $\begin{array}{l}\text { Improvement of arterial } \\
\text { plexus invasion on } \\
\mathrm{CT} \text { imaging }\end{array}$ & $\begin{array}{l}\text { Invasion to the extra } \\
\text { pancreatic nerve in } \\
\text { pathological diagnosis }\end{array}$ & $\begin{array}{l}\text { Residual } \\
\text { tumor }\end{array}$ & $\begin{array}{l}\text { Outcome } \\
\text { from IT (month) }\end{array}$ \\
\hline 1 & $\mathrm{~Pb}$ & $\mathrm{Ce}$ & DP-CAR & $\mathrm{Ce}$ & Partial & PLce $(+)$ & R1 & $44 \mathrm{DRD}$ \\
\hline 2 & $\mathrm{~Pb}$ & GDA & DP-CAR & $\mathrm{Ce}$ & Partial & PLce, cha $(+)$ & RO & $54 \mathrm{AFD}$ \\
\hline 3 & $\mathrm{~Pb}$ & GDA & DP-CAR & $\mathrm{Ce}, \mathrm{PV}$ & Partial & $\mathrm{PL}(-)$ & Ro & $92 \mathrm{AFD}$ \\
\hline 4 & $\mathrm{~Pb}$ & GDA & DP-CAR & $\mathrm{Ce}, \mathrm{PV}$ & Partial & PLcha, sma (+) & Ro & $55 \mathrm{AFD}$ \\
\hline 5 & $\mathrm{~Pb}$ & GDA & TP-CAR & $\mathrm{Ce}, \mathrm{GDA}, \mathrm{PV}$ & Partial & PLce, sma (+) & Ro & $67 \mathrm{AFD}$ \\
\hline 6 & $\mathrm{~Pb}$ & SMA & DP-CAR & $\mathrm{Ce}, \mathrm{PV}$ & Partial & PLspa (+) & Ro & $91 \mathrm{AFD}$ \\
\hline 7 & $\mathrm{Ph}$ & $\mathrm{CHA}$ & SSPPD & PV & Completely & $\mathrm{PL}(-)$ & Ro & 63 DRD \\
\hline 8 & $\mathrm{Ph}$ & $\mathrm{CHA}, \mathrm{PHA}$ & SSPPD & CHA, PHA, RHA, PV & Partial & $\mathrm{PL}(-)$ & Ro & 31 ARD \\
\hline 9 & $\mathrm{Ph}$ & $\mathrm{CHA}$ & SSPPD & CHA, PV & Partial & PLcha (+) & RO & $18 \mathrm{DRD}$ \\
\hline 10 & $\mathrm{~Pb}$ & SMA & DP-CAR & $\mathrm{Ce}, \mathrm{PV}$ & Completely & PLce $(+)$ & Ro & $32 \mathrm{AFD}$ \\
\hline 11 & $\mathrm{~Pb}$ & SMA & DP-CAR & $\mathrm{Ce}, \mathrm{PV}$ & Completely & PLce $(+)$ & Ro & 15 ARD \\
\hline 12 & $\mathrm{Ph}$ & $\mathrm{CHA}$ & SSPPD & CHA, LHA, MHA, PV & Partial & PLcha (+) & RO & $11 \mathrm{AFD}$ \\
\hline
\end{tabular}

Ce ceriac artery, GDA gastro-duodenal artery, CHA common hepatic artery, PHA proper hepatic artery, RHA right hepatic artery, LHA left hepatic artery, MHA middle hepatic artery, $P V$ portal vein, $A F D$ alive free of disease, $A R D$ alive with recurrent disease, $D R D$ dead of recurrent disease

pathological findings after resection showed tumor cells remaining around the celiac artery discontinuously (Fig. 2).

\section{Clinical factors associated with overall survival}

Overall survival of all patients is shown in Fig. 3. The median follow-up time from initial treatment in all 18 patients was 39 months, and the 5-year survival rate was $60.3 \%$.
The survival from initial treatment was significantly worse for patients with metastatic disease than for patients with locally advanced disease $(P=0.006)$ (Fig. 4).

Survival from initial treatment was much better for patients with $\mathrm{R} 0$ resection than for patients with $\mathrm{R} 1 / 2$ resection $(P=0.002)$ (Fig. 5).

Examining the pathology, there was a significant difference in the prognosis between Evans grade I-IIa and
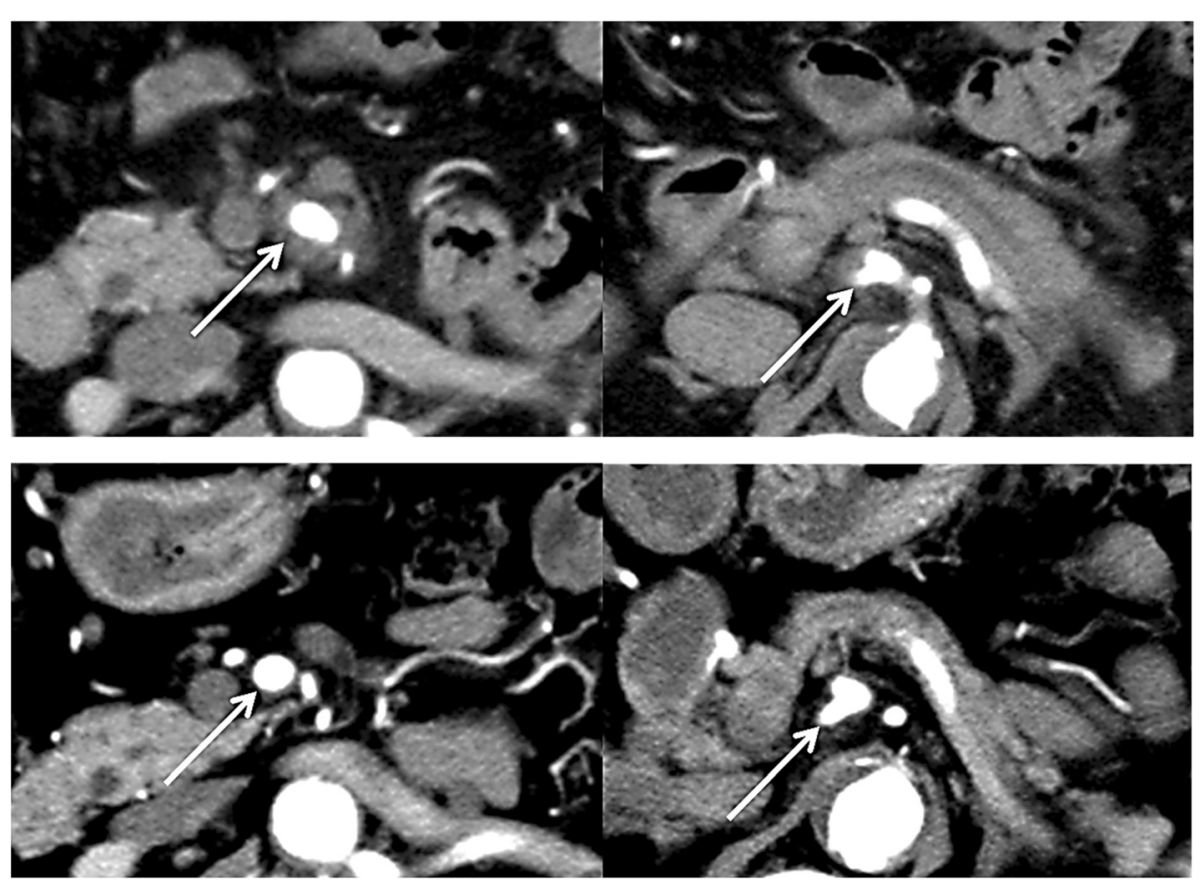

Fig. 1 CT scan shows tumor invading to the SMA and CA (above). CT scan shows the tumor decreased by chemotherapy (below). White arrows indicate tumor invasion to the SMA or CA 


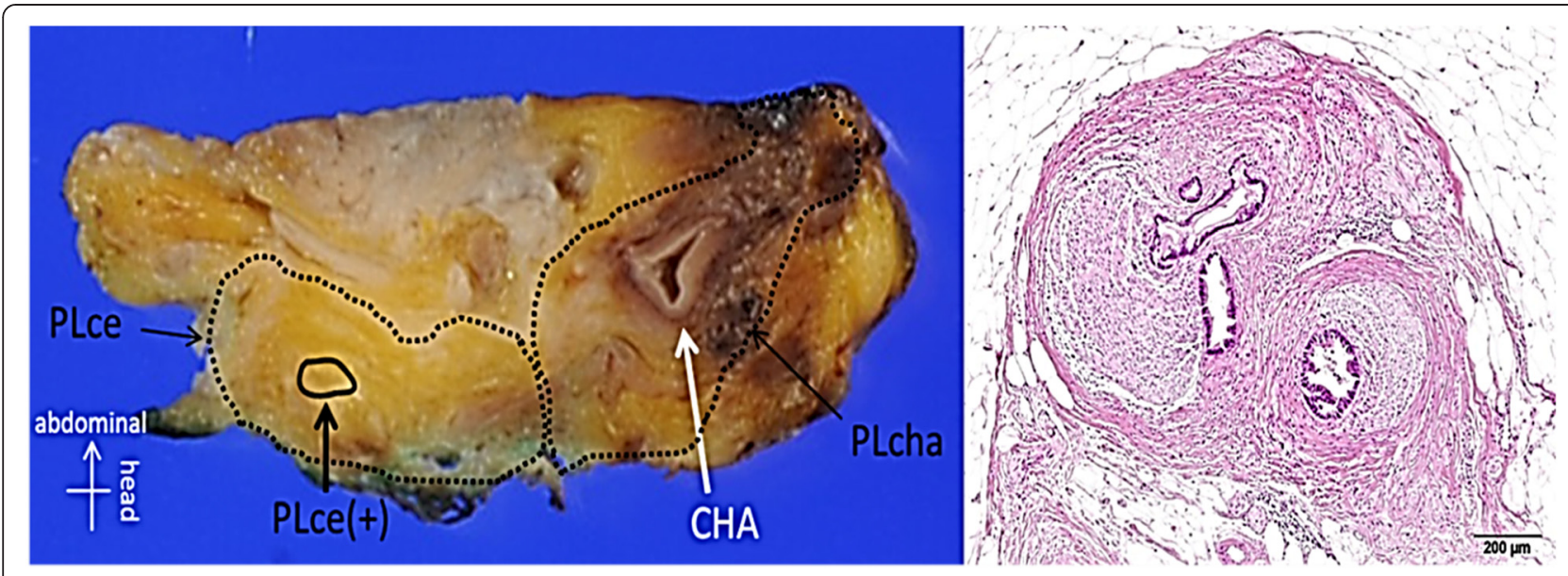

Fig. 2 Histopathological mapping on the macroscopic section at the plexus around the CA, indicating residual cancer cells at the plexus around the CA (left, area encircled with solid line). hematoxylin-eosin staining showed the residual cancer cells present at the area encircled with the broken line (right)

grade IIb-IV ( $P=0.046)$ (Fig. 6). However, there was no significant difference in the prognosis between patients with and without plexus invasion $(P=0.750)$.

\section{Discussion}

The prognosis of patients with unresectable pancreatic cancer is extremely poor, and development of new anti-cancer drugs including molecular target inhibitors has been in progress. Among clinical trials worldwide [11-17], several anti-cancer agents have been reported to have better response rates and 2-year survival rates with chemotherapy alone (41.9-81.3 \% and 23.5-27.1\%, respectively) $[18,19]$. However, there have been few cases with 5 -year survival treated by chemoradiotherapy alone. Therefore, we have performed adjuvant surgery for selected cases with a long-term favorable response to chemoradiotherapy since 2006. Satoi et al. reported, in their Project01 study by the Japanese Society of HepatoBiliary-Pancreatic Surgery, that the overall survival rate from the initial treatment with adjuvant surgery was higher than that with non-surgical anti-cancer treatment in patients who received chemoradiation therapy for more than 8 months [20]. Deyali reported in their series of 20 patients who were surgically resected after neoadjuvant chemotherapy that there was a correlation between patient prognosis and Evans grade between Evans grade I-IIb and grade III-IV [8]. Although the preoperative factors that could predict patient prognosis were not

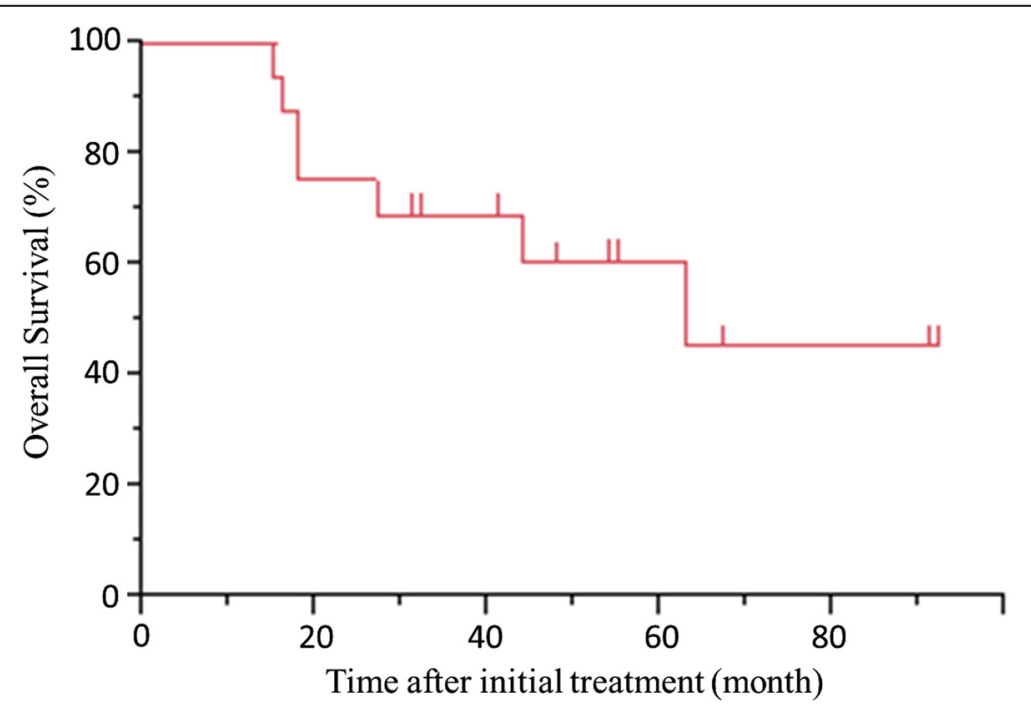

Fig. 3 Overall survival from initial treatment of all patients. The 5 -year survival rate is $60.3 \%$. The median follow-up time from initial treatment is 39 months (range: 11-92 months) 


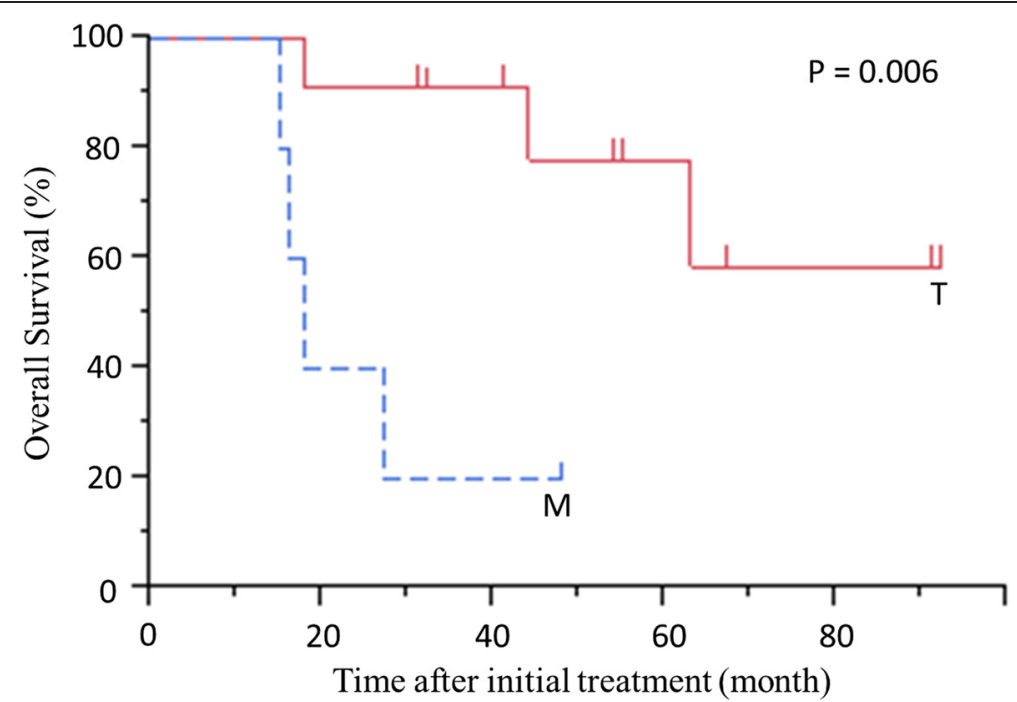

Fig. 4 Overall survival beginning at initial treatment in patients with locally advanced disease (T) or metastatic disease (M) as the reason for initial unresectability. $P=0.006$

identified, it is of great interest to predict the timing of the surgical intervention by preoperative biomarkers, because it is not clear whether the operation is truly good for patients with a good response to chemotherapy. A further prospective study is warranted in the future.

In the present series of 18 cases, the 5-year survival rate from initial treatment was $60.3 \%$, and there were 2 cases who survived more than 5 years after the operation. Surgical margin-negative was a significant predictor of a good prognosis. The period of preoperative treatment of these patients other than the R0 operation was 5-11 months, but it is unclear whether R0 resection is possible if the preoperative treatment period is increased.
Considering patients with $\mathrm{R} 0$ resection, the $\mathrm{R} 0$ rate of patients with distant metastasis was $60 \%(3 / 5)$ and that with locally advanced disease was $92 \%(12 / 13)$. Therefore, adjuvant surgery might be appropriate for patients with locally advanced pancreatic cancer, and it is important to achieve R0 resection. For hepatic metastasis, only one patient underwent concomitant hepatic resection with no pathological finding of malignancy. Other two patients did not undergo hepatic resection because of no findings of hepatic metastasis in CT before surgery. In terms of postoperative disease prognosis for the three patients with initial hepatic metastasis, one patient who underwent hepatic resection with no pathological

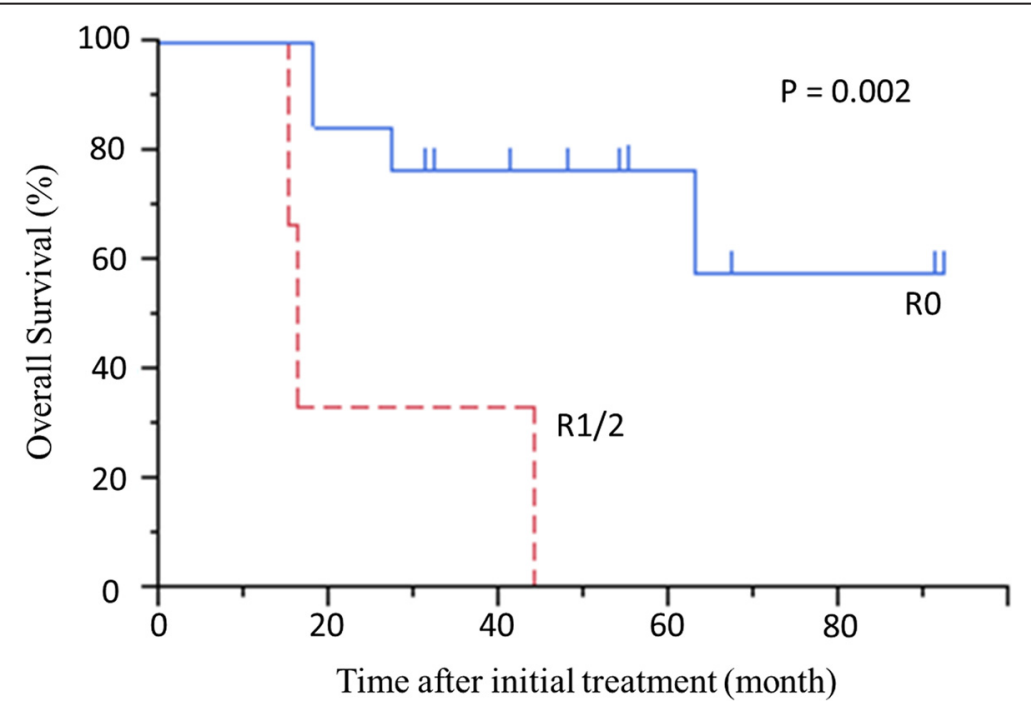

Fig. 5 Overall survival beginning at initial treatment in patients with $\mathrm{R} 0$ resection or R1/2 resection. $P=0.002$ 


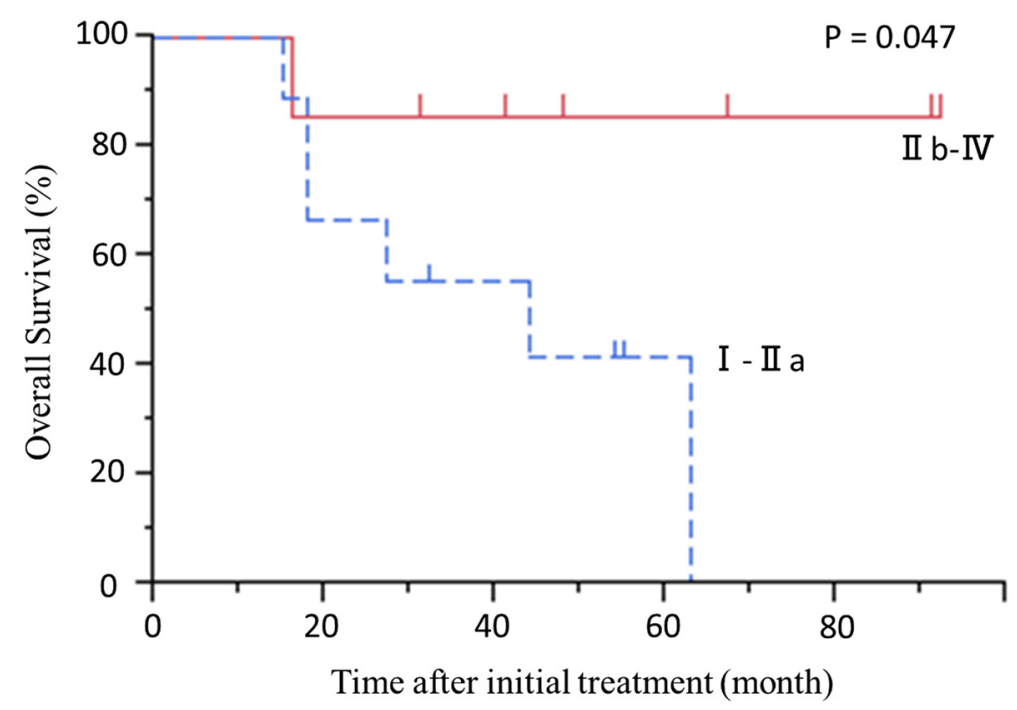

Fig. 6 Overall survival beginning at initial treatment in patients with Evans grade I-Ila or grade IIb-IV. $P=0.047$

evidences for malignancy died of para-aortic lymph node recurrence in 21 months after surgery. Other two patients without hepatic tumors after chemoradiotherapy developed multiple liver metastases including initial site in 4 months or 2 months, respectively, after primary site resection. The fact showed that they might have partial response for hepatic metastasis which was too small to detect in CT but not always showed complete response.

This was a retrospective analysis, so it is not clear whether a similar prognosis could be obtained if chemotherapy was continued in the patients with adjuvant surgery. Further study is needed to decide whether surgery should be performed after chemoradiotherapy in every case.

Regarding the surgical strategy following chemotherapy for initially unresectable pancreatic cancer, the extent of resection remains controversial. Whether the shrunken or arrested area which might have well responded to the chemoradiotherapy should also be included in the scheduled resection area is of the greatest interest. Although these kinds of surgical strategies concerning resection area after neoadjuvant chemoradiotherapy are well established in other cancers [21, 22], it is still controversial for initially unresectable pancreatic cancer. For pancreatic cancer, there have been only several reports of a small number of patients and limited cases with surgical procedures. Furthermore, there are few reports that suggest an appropriate treatment strategy, including the extent of resection for the initially unresectable cases after chemoradiotherapy.

In our department, we resect the area initially affected by tumor and regional major vessels even if tumor shrinkage was obtained and perineural invasion around the artery on imaging modality was improved by initial treatment. As shown in one characteristic case that had undergone DP-CAR with dissection of the initially suspected area with perivascular neural invasion, clear evidence was shown for the first time that the tumor did not shrink linearly or continuously from the tumor front but that part of it often remained discontinuously at the distal portion in the process of tumor regression by chemotherapy. It is unknown how remaining tumor cells in this way can affect recurrence or a patient's prognosis. Because of the good prognosis of patients with R0 resection, it has become clear from the pathological aspects that the operative procedure including fields initially affected by tumor should be concomitantly resected as much as possible, even if arterial resection and reconstruction are indicated.

There are some reports on the pros and cons of additional arterial resection, but satisfactory results have not been obtained for SMA resection, with a high rate of surgery-related mortality [23, 24]. Therefore, in our department, we perform additional arterial resection actively only for non-SMA cases. In the present study, 4 of the 18 patients underwent arterial resection and reconstruction, and R0 resection was achieved for all 4 patients. However, accumulation of further cases is needed to consider the long-term results for patients with arterial resection.

The limitations of this study were, firstly, the small number of the cases, secondly, selection vias with which we indicated surgery for patients who had favorable response to chemo(radio)therapy, and lastly, the retrospective nature of the study design. Therefore, statistical analysis in this study might be better interpreted as a trend instead of a statistical significance. And this study 
is the retrospective study for patients with unresectable pancreatic carcinoma. Future prospective study should be performed to clarify whether to continue chemotherapy or indicate radical R0 operation for patients with good response to chemo(radio)therapy.

\section{Conclusions}

In conclusion, in adjuvant surgery for patients with locally advanced pancreatic cancer, achieving $\mathrm{R} 0$ resection will improve the prognosis. Because of the potent residual cancer cells at the initial tumor front and the limitations of imaging diagnosis, it is necessary to consider how well the extent of resection is after a favorable response to chemo(radio)therapy.

\section{Competing interests}

The authors declare that they have no competing interests.

\section{Authors' contributions}

HT performed the statistical analysis and drafted the manuscript. TN and KO collected the patient's data. TS participated in the design of the study and performed the statistical analysis. TT and SH conceived of the study, participated in its design and coordination, and helped to draft the manuscript. All authors read and approved the final manuscript.

\section{Acknowledgements}

None

Received: 9 February 2015 Accepted: 3 June 2015

Published online: 26 June 2015

\section{References}

1. Warshaw AL, Fernandez-del CC. Pancreatic carcinoma. N Engl J Med. 1992;326:455-65.

2. Lionetto R, Pugliese V, Bruzzi P, Rosso R. No standard treatment is available for advanced pancreatic cancer. Eur J Cancer. 1995;31A(6):882-7.

3. Lygidakis NJ, Ziras FA, Kyparidou E, Parissis J, Papadopoulou P, Venetsanou B. Combined immunopharmaceutical therapy of patients with unresectable pancreatic carcinoma. Hepatogastroenterology. 1995;42(6):1039-52.

4. Sho M, Akahori T, Tanaka T, Kinoshita T, Tamamoto T, Nomi T, et al. Pathological and clinical impact of neoadjuvant chemoradiotherapy using full-dose gemcitabine and concurrent radiation for resectable pancreatic cancer. J Hepatobiliary Pancreat Sci. 2012;20(2):197-205.

5. Strobel O, Berens V, Hinz U, Hartwig W, Hackert T, Bergmann F, et al. Resection after neoadjuvant therapy for locally advanced, "unresectable" pancreatic cancer. Surgery. 2012;152:S33-42.

6. Kato K, Kondo S, Hirano S, Tanaka E, Shichinohe T, Tsuchikawa T, et al. Adjuvant surgical therapy for patients with initially-unresectable pancreatic cancer with long-term favorable responses to chemotherapy. J Hepatobiliary Pancreat Sci. 2011;18:712-6.

7. Deyali C, Matthew H, Asif R, Hua W, Alina C, Gauri R, et al. Perineural and intra-neural invasion in posttherapy pancreaticoduodenectomy specimens predicts poor prognosis in patients with pancreatic ductal adenocarcinoma. Am J Surg Pathol. 2012;36:409-17.

8. Deyali C, Matthew H, Asif R, Gauri R, Robert A, Hua W, et al. Histologic grading of the extent of residual carcinoma following neoadjuvant chemoradiation in pancreatic ductal adenocarcinoma. Cancer. 2012;118:3182-90.

9. Therasse P, Arbuck SG, Eisenhauer EA, Wanders J, Kaplan RS, Rubinstein L, et al. New guidelines to evaluate the response to treatment in solid tumors. European Organization for Research and Treatment of Cancer, National Cancer Institute of the United States, National Cancer Institute of Canada. J Natl Cancer Inst. 2000:92:205-16.

10. Evans DB, Rich TA, Byrd DR, Cleary KR, Connelly JH, Levin B, et al. Preoperative chemoradiation and pancreaticoduodenectomy for adenocarcinoma of the pancreas. Arch Surg. 1992;127:1335-9.
11. Childs DS, Moertel CG, Holbrook MA, Reitemeier RJ, Colby MY. Treatment of malignant neoplasms of the gastrointestinal tract with a combination of 5-fluorouracil and radiation. Radiology. 1965;84:843-8.

12. Hazel JJ, Thirwell MP, Huggins M, Maksymiuk A, MacFarlane JK. Multi-drug chemotherapy with and without radiation for carcinoma of the stomach and pancreas: a prospective randomized trial. J Can Assoc Radiol. 1981;32:164-5.

13. Klaassen DJ, Maclntyre JM, Catton GE, Engstrom PF, Moertel CG. Treatment of locally unresectable cancer of the stomach and pancreas: a randomized comparison of 5-fluorouracil alone with radiation plus concurrent and maintenance 5-fluorouracil—an Eastern Cooperative Oncology Group study. J Clin Oncol. 1985;3:373-8.

14. Gastrointestinal Tumor Study Group. Treatment of locally unresectable carcinoma of the pancreas: comparison of combined modality therapy (chemotherapy plus radiotherapy) to chemotherapy alone. J Natl Cancer Inst. 1988;80:751-5.

15. Saif MW. New developments in the treatment of pancreatic cancer. Highlights from the "44th ASCO Annual Meeting". Chicago, IL, USA. May 30-June 3, 2008. J Pancreas. 2008;9(4):391-7.

16. Chauffert B, Mornex F, Bonnetain F, Rougier P, Mariette C, Bouche O, et al. Phase III trial comparing an intensive induction chemoradiotherapy (60 Gy, infusional 5-FU and intermittent cisplatin) followed by maintenance gemcitabine with gemcitabine alone for locally advanced unresectable pancreatic cancer: definitive results of the 2000-01 FFCD/SFRO study. Ann Oncol. 2008; 19:1592-9.

17. Philip PA, Benedetti J, Corless CL, Wong R, O'Reilly EM, Flynn PJ, et al. Phase III study comparing gemcitabine plus cetuximab versus gemcitabine in patients with advanced pancreatic adenocarcinoma: Southwest Oncology Group-directed intergroup trial S0205. J Clin Oncol. 2010;28:3605-10.

18. Gillmore R, Laurence V, Raouf S, Tobias J, Blackman G, Meyer T, et al. Chemoradiotherapy with or without induction chemotherapy for locally advanced pancreatic cancer: a UK multi-institutional experience. Clin Oncol. 2010;22:564-9

19. Ogawa K, Ito Y, Hirokawa N, Shibuya K, Kokubo M, Ogo E, et al. Concurrent radiotherapy and gemcitabine for unresectable pancreatic adenocarcinoma: impact of adjuvant chemotherapy on survival. Int I Radiat Oncol Biol Phys. 2012;83(2):559-65.

20. Satoi S, Yamaue H, Kato K, Takahashi S, Hirono S, Takeda S, et al. Role of adjuvant surgery for patients with initially unresectable pancreatic cancer with a long-term favorable response to non-surgical anti-cancer treatments: results of a project study for pancreatic surgery by the Japanese Society of Hepato-Biliary-Pancreatic Surgery. J Hepatobiliary Pancreat Sci. 2013;20:590-600

21. McVeigh TP, Al-Azawi D, Kearney DE, Malone C, Sweeney KJ, Barry K, et al. Assessing the impact of neoadjuvant chemotherapy on the management of the breast and axilla in breast cancer. Clin Breast Cancer. 2013;14:20-5.

22. Marín C, Robles R, López Conesa A, Torres J, Flores DP, Parrilla P. Outcome of strict patient selection for surgical treatment of hepatic and pulmonary metastases from colorectal cancer. Dis Colon Rectum. 2013;56:43-50.

23. Nakao A, Takeda S, Inoue S, Nomoto S, Kanazumi N, Sugimoto H, et al. Indications and techniques of extended resection for pancreatic cancer. World J Surg. 2006;30:976-82.

24. Kitagawa H, Ohta T, Tajima H, Nakagawara H, Makino I, Takamura H, et al. En bloc resection including the superior mesenteric artery and vein of locally advanced pancreatic carcinomas. J Jpn Surg Soc. 2011;112(3):164-9. 